\title{
OSH RISK EVALUATION WITHIN AGRO-INDUSTRIAL PLANTS: THE CASE OF AN OLIVE MILL
}

\author{
Alexandros Sotirios ANIFANTIS, Simone PASCUZZI, Francesco SANTORO \\ Department of Agricultural and Environmental Science (DiSAAT), University of Bari Aldo Moro, ITALY \\ E-mail of corresponding author: francesco.santoro@uniba.it
}

Keywords: electromagnetic fields, olive mill, risk assessment, worker's health

\begin{abstract}
The risk related to the presence of low frequency electromagnetic fields can be a serious OSH risk within different kind of agro-industrial plants. In this paper, an experimental analysis aimed at measuring electromagnetic fields in the productive areas of an olive mill recently built in the Bari district was accomplished. The analysis' main goal was to assess the workers' health risk level due to the presence of extremely low frequency electromagnetic fields $(30 \div 300 \mathrm{~Hz})$ mainly due to the presence of electrical distribution lines and electric motors operating at a frequency of $50 \mathrm{~Hz}$ with reference to a specific type of processing plants, widely spread in the Apulia region. A first analysis of the spatial layout of the electric machine was carried out, referring to their duty cycles and to workers' tasks (taking into particular account the working places) identifying 12 most significant points in which the measurements of effective value of the electrical and magnetic field would have been carried out. Effective values of the electric field $\left(E_{R M S}\right)$ as well as effective values of the magnetic induction field $\left(B_{R M S}\right)$ measured in each testing point, were both significantly lower than the limit values required by law. Peak values of both the electric field $\left(E_{\text {peak }}\right)$ and the magnetic induction field $\left(B_{\text {peak }}\right)$ measured in the different testing points at the frequency of $50 \mathrm{~Hz}$, were lower than the corresponding effective values $E_{R M S}$ and $B_{R M S}$. The results of the tests highlight that, inside the analysed olive mill, in the period of its maximum productivity, risks for the workers' health related to their exposure to low frequency electric and magnetic fields are not relevant.
\end{abstract}

\section{INTRODUCTION}

Even if exposure to electromagnetic fields is not a new phenomenon, in the last decades the risk associated with the spread in the environment of man-made electromagnetic fields has been steadily increasing.

Everyone is exposed to a complex mix of weak electric and magnetic fields which originate from the generation and transmission of electricity, from the use of industrial equipment, from to telecommunications and broadcasting, etc. When the presence of an electromagnetic field affects a working place, workers' health and safety should be taken into proper account.

The Italian D.Lgs 81/2008, coordinated with Italian D.Lgs 106/2009 among the various risks for the health of the workers, includes the ones connected to the exposure to electromagnetic fields $(0 \mathrm{~Hz} \div 300 \mathrm{GHz})$ during the job.

Under a sanitary point of view, an individual dipped in an electromagnetic field interacts with it creating a physical coupling between his biological system and the field, which evidently produces a deviation from the conditions of electrical balance at molecular level.

The dielectric features of biological tissues and the electromagnetic field frequency affect the physical mechanisms of coupling between electromagnetic fields and biological organisms, therefore the reaction of a human body could very different if a worker is exposed to high frequency electromagnetic field generated by telecommunications and broadcasting systems (Pascuzzi S., Santoro F., 2015) or if the same worker is exposed to a low frequency electromagnetic field. 
Low-frequency electric fields influence the human body just as they influence any other material made up of charged particles. When electric fields act on conductive materials, they influence the distribution of electric charges at their surface. They cause current to flow through the body to the ground. Low-frequency magnetic fields induce circulating currents within the human body. The strength of these currents depends on the intensity of the outside magnetic field. If sufficiently large, these currents could cause stimulation of nerves and muscles or affect other biological processes.

Therefore, within the low frequency electromagnetic fields $(0 \div 10 \mathrm{kHz})$, a person's exposure is directly bound to the values of a few electrical characteristics which establish themselves as an effect of such fields, inside the human body: such characteristics, internal or primary, are essentially the intensity of the electrical field and above all the internal current density, defined as the current which passes through a unitary section perpendicular to its direction in a conductive volume such as the human body or a part of it. These primary characteristics, however, are difficult to measure in the real exposure conditions and, therefore, a person's exposure to the electrical and magnetic fields is obtained through easier to measure outside or derived characteristics: the effective values of the inductive electrical and magnetic fields in absence of the exposed body. From these derived characteristics, thought the evaluation of the exposure conditions and of the relative characteristic of the electromagnetic field, by mean of dosimetric models the primary ones are obtained.

This paper aims to highlight the results of a survey carried out in an olive-mill located in Corato town, in the north of Bari province chosen as it was considered representative of a widely spread type of such productive plants present in the Apulia region.

The authors have already studied problems regarding the impact of electromagnetic field on workers safety and health within agro industrial sectors; this paper aims to be a further contribution in this topic.

\section{MATERIALS AND METHODS}

The considered olive-mill was organized with three independent production lines similar each other (defoliator, washer, crusher, malaxer and decanter) and, near the area occupied by one of the decanter, was located a small table with some chairs which allowed the customers to rest and manage their business. It was a family-driven business activity; during the main olive oil production period, all the three production lines work at their maximum capacity, the work is continuous over 24 hours and organized with three 8hour shifts.

A first analysis of the spatial layout of the electric machine was carried out, referring to their duty cycles and to workers' tasks (taking into particular account the working places). This preliminary analysis identified the 12 most significant points in which the measurements of effective value of the electrical and magnetic field would have been carried out. These points are essentially located near the electric motors which drive each operating machine (Figure 1).

At these points, for the duration of a full work shift, measurements according to the CEI 211-6 Italian standard instructions for reactive near-field region were carried out.

At the height of $150 \mathrm{~cm}$ above the floor level, which can be considered the mean height of the human thorax, were recorded both components of electrical and magnetic fields: in the reactive near-field region, in fact, no correlation between electrical and magnetic field 
exists since the first depends on the voltage present in the system or in the equipment which produces such field, the second depends on the currents in them circulating, being tensions and currents completely independent.

The low frequency electric and magnetic fields measurement system used was made by a PMM mod. EHP-50C analyser which complies both to Italian 10/09/98 D.M. 381 and to Italian 07/08/03 DPCM positioned on a non-magnetic tripod (Figure 2).

The electric and magnetic field in the frequency range $5 \div 100 \mathrm{~Hz}$, with a $0.25 \mathrm{~Hz}$ scanning interval, for the three components of a global orthogonal Cartesian reference system has been measured in each of the testing points.

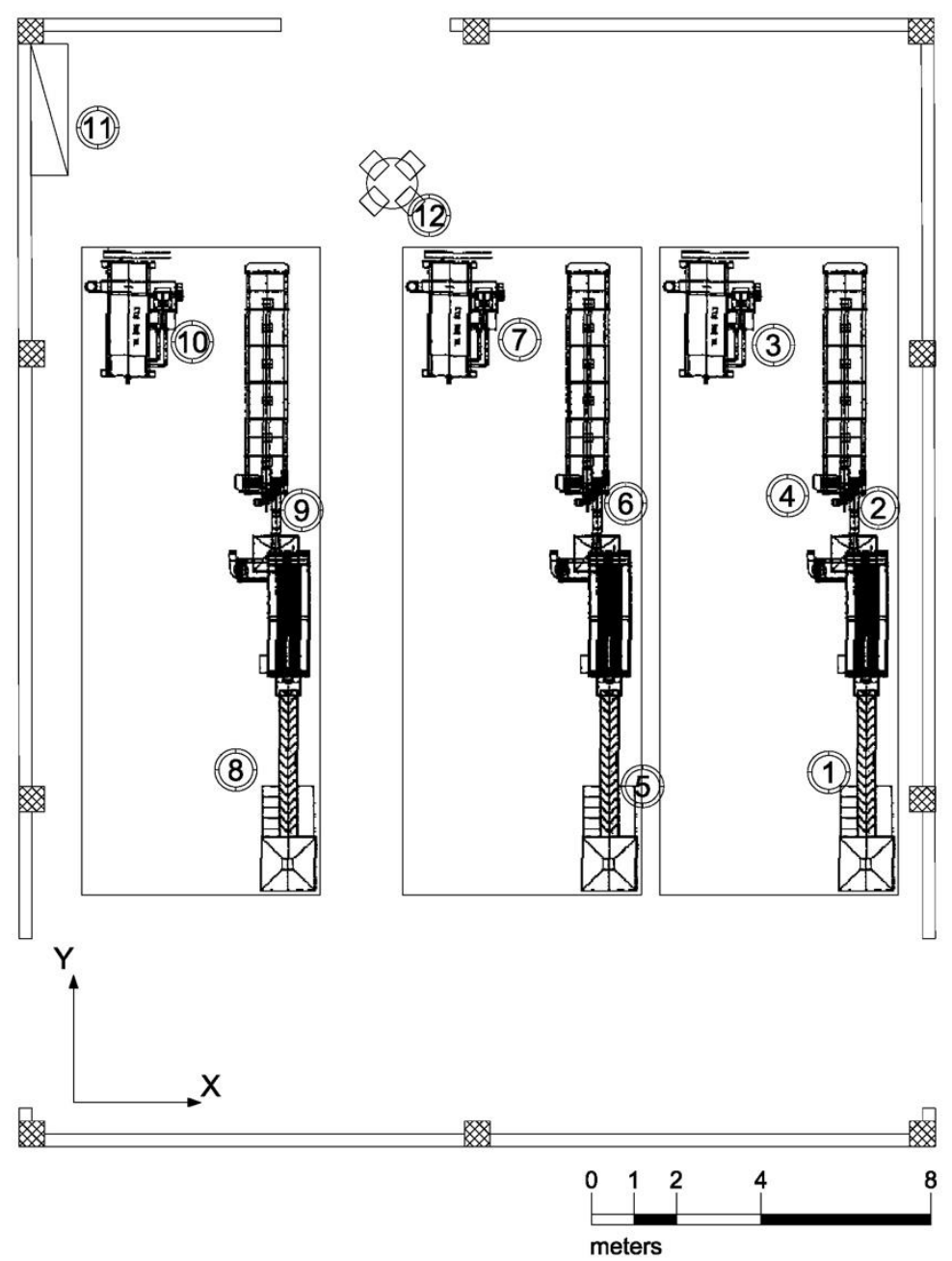

Figure 1. Oil-mill lay-out with indication of electromagnetic field measurement points. 


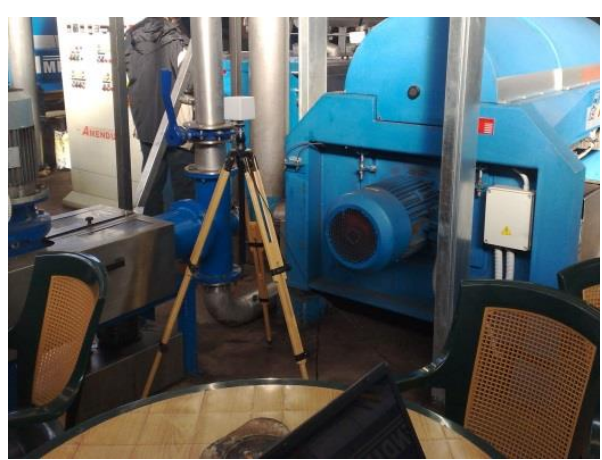

Figure 2. Measurement system standing at test point number 12.

\section{RESULTS AND DISCUSSION}

Effective values of the electric field (ERMS) as well as effective values of the magnetic induction field (BRMS) measured in each testing point, whilst taking into account the components along each of the three axes and the whole $5 \div 100 \mathrm{~Hz}$ frequency range examined were obtained using the following two equations:

$$
\begin{aligned}
& E_{R M S}=\sqrt{\frac{1}{n} \cdot \sum_{i=1}^{n}\left(E_{x i}^{2}+E_{y i}^{2}+E_{z i}^{2}\right)} \\
& B_{R M S}=\sqrt{\frac{1}{n} \cdot \sum_{i=1}^{n}\left(B_{x i}^{2}+B_{y i}^{2}+B_{z i}^{2}\right)}
\end{aligned}
$$

Both effective values of the electric field (ERMS) and effective values of the magnetic induction field (BRMS) were considerably lower than the action values given in the laws for the protection of the health and safety of workers currently in force in Italy as reported in Figure 3

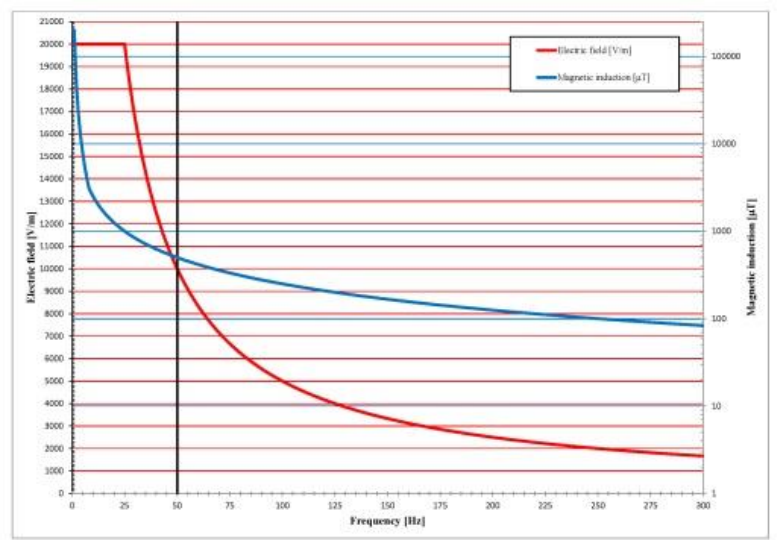

Figure 3. Effective action values of electrical field $E_{R M S}$ and $B_{R M S}$.

Furthermore, peak values of both the electric field (EPEAK) and the magnetic induction field (BPEAK) measured in the different testing points at the frequency of $50 \mathrm{~Hz}$, were lower than the corresponding effective values ERMS and BRMS. 


\section{CONCLUSIONS}

The results of the tests carried out confirms other previous surveys carried out in other Apulian olive-mills. Once again, the results highlight that, also inside the analysed olive mill in this paper, in the period of its maximum productivity, risks for the workers' health related to their exposure to low frequency electric and magnetic fields are not relevant.

It can also be generally said that the electrical field turns out significant only if there are machines, conductors or, generally speaking, tools which require high voltage to work and that the magnetic induction field produced, instead, by most electrical tools, has a low coupling with human bodies and turns out only in a very close region around its source.

\section{REFERENCES}

Bevitori P. (2007). Inquinamento da campi elettrici, magnetici ed elettromagnetici. Maggioli Editore

CEI 211-6 (2001). Guide for the measurement and the evaluation of electric and magnetic fields in the frequency range $0 \mathrm{~Hz}-10 \mathrm{kHz}$, with reference to the human exposure.

Italian DLgs n. 81 (2008). Testo unico sulla salute e sicurezza sul lavoro. GU 101 30/04/2008

ICNIRP (1998). Guidelines for limiting exposure to time-varying electric, magnetic and electromagnetic fields (up to $300 \mathrm{GHz}$ ). Health Physics, 74(4), 494-522.

ICNIRP (2010). Guidelines for limiting exposure to time-varying electric and magnetic fields ( $1 \mathrm{~Hz}-100$ kHz). Health Physics 99(6), 818-836

Pascuzzi S., Santoro F. (2015). Exposure of farm workers to electromagnetic radiation from cellular network radio base stations situated on rural agricultural land. International Journal of Occupational Safety and Ergonomics, 21(3), 351-358

SCENIHR (2015). Potential health effects of exposure to electromagnetic fields (EMF). http://ec.europa.eu/health/scientific_committees/emerging/docs/scenihr_o_041.pdf. 15/08/2015. 